Boise State University

ScholarWorks

3-9-2009

Ferroelectric Phase Transitions in ThreeComponent Short-Period Superlattices Studied by Ultraviolet Raman Spectroscopy

Dmitri Tenne

Boise State University

H. N. Lee

Oak Ridge National Laboratory

R. S. Katiyar

University of Puerto Rico

X.X.Xi

Pennsylvania State University 
This is an author-produced, peer-reviewed version of this article. The final, definitive version of this document can be found online

at Journal of Applied Physics, published by American Institute of Physics. Copyright restrictions may apply. doi: 10.1063/1.3087611

\title{
Ferroelectric phase transitions in three-component short-period superlattices studied by ultraviolet Raman spectroscopy
}

\author{
D. A. Tenne* \\ Department of Physics, Boise State University, Boise, ID 83725-1570 \\ H. N. Lee \\ Materials Science and Technology Division, \\ Oak Ridge National Laboratory, Oak Ridge, TN 37831-6030, USA \\ R. S. Katiyar \\ Department of Physics, University of Puerto Rico, \\ San Juan, 00931-3343, Puerto Rico \\ X. X. Xi \\ Department of Physics, Department of Materials Science \\ and Engineering, and Materials Research Institute, \\ the Pennsylvania State University, University Park, PA 16802
}

(Dated: January 19, 2009)

\begin{abstract}
Vibrational spectra of three-component $\mathrm{BaTiO}_{3} / \mathrm{SrTiO}_{3} / \mathrm{CaTiO}_{3}$ short-period superlattices grown by pulsed laser deposition with atomic-layer control have been investigated by ultraviolet Raman spectroscopy. Monitoring the intensity of the first-order phonon peaks in Raman spectra as a function of temperature allowed determination of the ferroelectric phase transition temperature, $T_{c}$. Raman spectra indicate that all superlattices remain in the tetragonal ferroelectric phase with out-of-plane polarization in the entire temperature range below $T_{c}$. The dependence of $T_{c}$ on the relative thicknesses of ferroelectric $\left(\mathrm{BaTiO}_{3}\right)$ to non-ferroelectric materials $\left(\mathrm{SrTiO}_{3}\right.$ and $\left.\mathrm{CaTiO}_{3}\right)$ has been studied. The highest $T_{c}$ was found in superlattices having the largest relative amount of $\mathrm{BaTiO}_{3}$, provided that the superlattice maintains its coherency with the substrate. Strain relaxation leads to a significant decrease in the ferroelectric phase transition temperature.

PACS numbers: 77.84.Dy, 77.80.Bh, 78.30.-j, 63.22.+m
\end{abstract}


This is an author-produced, peer-reviewed version of this article. The final, definitive version of this document can be found online

at Journal of Applied Physics, published by American Institute of Physics. Copyright restrictions may apply. doi: 10.1063/1.3087611

\section{INTRODUCTION}

Recent advances in epitaxial technology made possible the synthesis of multilayer oxide structures with monolayer-precision control of thicknesses and atomically flat interfaces. ${ }^{1-3}$ This opened a way for synthesis of artificial materials, such as ultrathin films or nanoscale ferroelectric heterostructures. Reduction of the structural dimensions to the nanometer scale gives rise to physical phenomena and properties dramatically different from those of homogeneous bulk ferroelectrics. ${ }^{4-9}$ A particular type of ferroelectric nanostructures that has recently received increased attention is ferroelectric superlattice (SL), i.e. the periodic structure containing alternating layers of different materials. Several groups have grown high quality ferroelectric superlattices with nearly atomically sharp interfaces in few perovskite systems, such as $\mathrm{BaTiO}_{3} / \mathrm{SrTiO}_{3}, 1,10,11, \mathrm{BaTiO}_{3} / \mathrm{PbTiO}_{3},{ }^{12} \mathrm{PbTiO}_{3} / \mathrm{SrTiO}_{3},{ }^{9,13-15}$ $\mathrm{KNbO}_{3} / \mathrm{KTaO}_{3},{ }^{16,17} \mathrm{SrTiO}_{3} / \mathrm{BaZrO}_{3},{ }^{18}$ and more complex three-component superlattices $\mathrm{CaTiO}_{3} / \mathrm{BaTiO}_{3} / \mathrm{SrTiO}_{3} .^{2,19}$ The properties of such structures are not just simple combination of those known for constituent bulk materials. In particular, it was described theoretically ${ }^{20-22}$ and observed experimentally, ${ }^{2,11}$ that remanent polarization in SLs can be enhanced above the value of that in bulk constituents.

One of the major factors affecting the behavior of nanoscale ferroelectric thin films and multilayer structures is epitaxial strain. ${ }^{23}$ Recent demonstrations of huge strain effect on ferroelectric properties include dramatic enhancement of ferroelectricity in $\mathrm{BaTiO}_{3}$ films (increase of the ferroelectric phase transition temperature $T_{c}$ by hundreds of degrees and polarization enhancement by $250 \%$ compared to bulk $\left.\mathrm{BaTiO}_{3}\right)^{24}$ and observation of roomtemperature ferroelectricity in strained films of $\mathrm{SrTiO}_{3},{ }^{25}$ a material, which is not ferroelectric in its bulk form at any temperature. Size effect is another factor strongly influencing the properties of ferroelectric nanostructures, and the issue of a critical size for ferroelectricity is actively discussed. , $7,8,26$ For a long time it was believed that ferroelectricity was suppressed in small particles and thin films, ${ }^{27}$ and there was a critical size in order of few tens of nanometers below which a spontaneous polarization cannot be sustained in a material. Later experiments identified ferroelectric state in $4 \mathrm{~nm}$-thick perovskite oxide films, ${ }^{28}$ and recent theoretical work ${ }^{29,30}$ has indicated that the critical size is orders of magnitude smaller than previously thought. Very recent results on ultrathin $\mathrm{PbTiO}_{3}$ films ${ }^{31-34}$ and superlattices ${ }^{2,9,14,15,35}$ provided the experimental evidence that ferroelectricity persists down to 
This is an author-produced, peer-reviewed version of this article. The final, definitive version of this document can be found online

at Journal of Applied Physics, published by American Institute of Physics. Copyright restrictions may apply. doi: 10.1063/1.3087611

vanishingly small sizes, it can exist in superlattices containing only one unit cell thick layer of ferroelectric $\left(\mathrm{PbTiO}_{3}\right.$ or $\left.\mathrm{BaTiO}_{3}\right)$ embedded in much thicker non-ferroelectric $\mathrm{SrTiO}_{3}$. These studies revealed that the issue of critical size is very complex, and electrical and mechanical boundary conditions play essential role in nanoscale ferroelectricity.

Recently, three-component superlattices consisting of ferroelectric $\mathrm{BaTiO}_{3}$, and nonferroelectric titanates, $\mathrm{CaTiO}_{3}$ and $\mathrm{SrTiO}_{3}$ fabricated by molecular beam epitaxy ${ }^{19}$ and atomic-layer precision pulsed laser deposition ${ }^{2}$ were studied. Using three different materials allows building a structure with broken inversion symmetry. The resulting asymmetry of the ferroelectric double-well potential in such a structure suggests a possible appearance of effective permanent bias fields, predicted theoretically ${ }^{36}$ and observed experimentally. ${ }^{19}$ Use of $\mathrm{CaTiO}_{3}$ (bulk pseudocubic lattice constant $a=3.83 \AA$ ) and $\mathrm{BaTiO}_{3}(a=3.992 \AA)$ ) in combination with $\mathrm{SrTiO}_{3}$ can result in a superlattice growing commensurately on $\mathrm{SrTiO}_{3}$ substrate $(a=3.905 \AA)$. In such a superlattice, the constituent $\mathrm{BaTiO}_{3}$ and $\mathrm{CaTiO}_{3}$ layers are subject to significant epitaxial strain of opposite signs. Due to the strong coupling between strain and polarization in ferroelectrics, this can result in substantial enhancement of the polarization relative to that of the bulk constituents. Breakdown of inversion symmetry resulting in asymmetric polarization allows for and an extra degree of freedom for optimizing the ferroelectric properties in these superlattices. Recently, Nakhmanson, Rabe and Vanderbilt developed a model of polarization in such multicomponent superlattices, which can be used to optimize the arrangement of individual $\mathrm{CaTiO}_{3}, \mathrm{SrTiO}_{3}$, and $\mathrm{BaTiO}_{3}$ layers in a superlattice, predicting structures with the highest possible polarization and a low in-plane lattice constant mismatch with the substrate. ${ }^{22} \mathrm{H}$. N. Lee et al. ${ }^{2}$ reported the growth of $\mathrm{CaTiO}_{3} / \mathrm{BaTiO}_{3} / \mathrm{SrTiO}_{3}$ superlattices, demonstrated their excellent structural quality and polarization enhancement. However, these studies were all carried out at room temperature, and the phase transition behavior, in particular, the $T_{c}$, has not been determined. Lattice vibrations in these three-component superlattices have not been studies so far.

Vibrational (Raman and infrared) spectroscopies can provide valuable information for understanding the behavior of nanoscale ferroelectrics, as the lattice dynamics determines the fundamental ferroelectric properties. ${ }^{37}$ The phonon frequencies and their Raman activity are sensitive to the phase transitions in ferroelectrics. However, conventional Raman measurements of ultrathin ferroelectric films and nanostructures in the visible range are difficult or practically impossible because of the film transparence and small thickness, which 
This is an author-produced, peer-reviewed version of this article. The final, definitive version of this document can be found online

at Journal of Applied Physics, published by American Institute of Physics. Copyright restrictions may apply. doi: 10.1063/1.3087611

leads to extremely weak Raman signals from nanometer-thick films and the dominance of a substrate signal in the spectra. Using of ultraviolet (UV) excitation for Raman experiments allows to overcome these difficulties. ${ }^{35}$ For UV excitation, the photon energy is above the bandgap of ferroelectric oxides such as $\mathrm{SrTiO}_{3}, \mathrm{BaTiO}_{3}$, which leads to a much stronger absorption and a greatly reduced penetration depth, thus preventing light from entering the substrate. Therefore, the phonons of ultrathin films and superlattices can be observed in the UV Raman spectra without overwhelming substrate signal. ${ }^{35} \mathrm{UV}$ Raman spectroscopy was demonstrated to be an effective technique to determine the phase transition temperature $T_{c}$ in ferroelectric ultrathin films and superlattices. ${ }^{35}$ In the present paper we apply this technique to study the lattice dynamics in three-component $\mathrm{CaTiO}_{3} / \mathrm{BaTiO}_{3} / \mathrm{SrTiO}_{3}$ superlattices. We focus on the temperature evolution of the vibrational spectra and the phase transition behavior of these superlattices.

\section{EXPERIMENTAL DETAILS}

We performed UV Raman experiments on a series of three-component $\mathrm{SrTiO}_{3} / \mathrm{BaTiO}_{3} / \mathrm{CaTiO}_{3}$ SLs with atomically smooth interfaces grown on $\mathrm{SrTiO}_{3}$ (001)-oriented substrates with $\mathrm{SrRuO}_{3}$ buffer layers by pulsed laser deposition with atomic-layer precision. The growth details have been described elsewhere. ${ }^{2}$ The growth was monitored by reflection high energy electron diffraction. The samples were characterized by atomic force microscopy (AFM), high resolution scanning transmission electron microscopy (STEM), X-ray diffraction (XRD), and reciprocal space mapping (RSM). ${ }^{2}$ AFM and STEM data demonstrated high structural quality of both the SLs and underlying $\mathrm{SrRuO}_{3}$ layers: the single-monolayer terrace steps on the $\mathrm{SrRuO}_{3}$ surface and on the top SL surface after the growth, and abrupt interfaces between the layers. The $\theta-2 \theta$ XRD scans show excellent diffraction pattern with all the superlattice peaks present, thus confirming the designed periodicity of the SLs. (See Ref. 2 for the details of structural characterization)

Parameters of the superlattice samples studied are listed in Table I. All the SLs studied had the total thickness of $200 \mathrm{~nm}$. RSM data were used to examine the strain state of the SLs and $\mathrm{SrRuO}_{3}$ buffer layers. The thickness of the $\mathrm{SrRuO}_{3}$ buffer layers was $4-16 \mathrm{~nm}$, and the films were grown at the conditions corresponding to the stability range of $\mathrm{SrRuO}_{3}$ films. ${ }^{2,38}$ Such thin $\mathrm{SrRuO}_{3}$ layers are usually grown coherently on $\mathrm{SrTiO}_{3}$ substrates. ${ }^{38,39}$ 
This is an author-produced, peer-reviewed version of this article. The final, definitive version of this document can be found online

at Journal of Applied Physics, published by American Institute of Physics. Copyright restrictions may apply. doi: 10.1063/1.3087611

Indeed, according to the RSM data of Lee et al., the $\mathrm{SrRuO}_{3}$ layers in the samples studied are commensurate with $\mathrm{SrTiO}_{3}$ substrates. The strain relaxation data for superlattices studied are given in the Table I ( $0 \%$ means fully strained, commensurate SL having the same inplane lattice parameter as $\mathrm{SrTiO}_{3}$ substrate). A $200 \mathrm{~nm}$-thick $\mathrm{BaTiO}_{3}$ film was also studied for comparison. This film is fully relaxed due to large thickness. From RSM data it follows that the $\mathrm{SLs}$ with $\mathrm{BaTiO}_{3}$ layer thickness not exceeding the combined thickness of $\mathrm{SrTiO}_{3}$ and $\mathrm{CaTiO}_{3}$ layers are coherently strained; the $\mathrm{SLs}$ with thicker $\mathrm{BaTiO}_{3}$ layers are partially relaxed. ${ }^{2}$

Raman spectra were recorded using a UV-optimized Jobin Yvon T64000 triple spectrometer equipped with a liquid-nitrogen-cooled multichannel coupled-charge-device detector. Spectra were recorded in backscattering geometry in the temperature range 30-600 K. For low-temperature measurements, the samples were attached to the cold finger inside a closed cycle helium cryostat. For measurements at elevated temperatures (above $300 \mathrm{~K}$ ) an evacuated heater stage was employed. The $325 \mathrm{~nm}$ line of He-Cd laser was used for excitation.

\section{RESULTS AND DISCUSSION}

The low temperature $(30 \mathrm{~K})$ UV Raman spectra of $\mathrm{CaTiO}_{3} / \mathrm{BaTiO}_{3} / \mathrm{SrTiO}_{3} \mathrm{SLs}$ and a $200 \mathrm{~nm}$-thick $\mathrm{BaTiO}_{3}$ film are shown in Fig. 1. All the SLs studied are ferroelectric at low temperatures, and the first-order Raman peaks of superlattice phonons are seen in the spectra. For identification of the observed optical phonon peaks in the UV Raman spectra of the superlattices, we compared them with the spectra of bulk and thin film $\mathrm{BaTiO}_{3}{ }^{40,41}$, $\mathrm{SrTiO}_{3}{ }^{42-44}, \mathrm{CaTiO}_{3}{ }^{45,46}$, as well as binary $\mathrm{SLs} \mathrm{BaTiO}_{3} / \mathrm{SrTiO}_{3} \cdot{ }^{35}$ The peaks at about 290 $\mathrm{cm}^{-1}$ in the spectra of fully or nearly fully strained SLs (spectra 2-5 in Fig.1) have similar positions and shapes to the $\mathrm{TO}_{2}$ modes of $A_{1}$ symmetry of $\mathrm{BaTiO}_{3}$ in the tetragonal phase, although there is probably a contribution of $\mathrm{TO}_{3}$ and $\mathrm{LO}_{2}$ modes observed in the spectra of bulk $\mathrm{BaTiO}_{3}{ }^{40}$ at $304-308 \mathrm{~cm}^{-1}$. Similar features are characteristic for the spectra of bulk and thin film $\mathrm{BaTiO}_{3}{ }^{40,41}$, and $\mathrm{BaTiO}_{3} / \mathrm{SrTiO}_{3} \mathrm{SLs}^{35}$ Therefore we assign them to the $\mathrm{BaTiO}_{3}$ phonons (note that this feature is almost absent in the spectra of the sample S1B1C1 having the smallest $\mathrm{BaTiO}_{3}$ content) and conclude that $\mathrm{BaTiO}_{3}$ layers are likely tetragonal in these strained SLs.

The line at about $180 \mathrm{~cm}^{-1}$ in the SL spectra, also observed in $\mathrm{BaTiO}_{3} / \mathrm{SrTiO}_{3} \mathrm{SLs}^{35}$ 
This is an author-produced, peer-reviewed version of this article. The final, definitive version of this document can be found online

at Journal of Applied Physics, published by American Institute of Physics. Copyright restrictions may apply. doi: 10.1063/1.3087611

is attributed to the first-order Raman scattering by $\mathrm{SrTiO}_{3}$-like $\mathrm{TO}_{2}$ phonons in SLs. Its position corresponds closely to that of bulk and thin-film $\mathrm{SrTiO}_{3},{ }^{43,44}$ but it is not from the substrate, because in bulk $\mathrm{SrTiO}_{3}$ the first-order Raman lines are symmetry-forbidden, while the SLs are ferroelectric, and the symmetry is broken. Moreover, due to the absorption of UV light by rather thick superlattices, neither the substrate nor $\mathrm{SrRuO}_{3}$ buffer layer contribution is present in the spectra. $\mathrm{BaTiO}_{3}$ also has a $\mathrm{TO}_{1}$ mode of $A_{1}$ symmetry at about the same position $\left(177 \mathrm{~cm}^{-1}\right),{ }^{40}$ and $\mathrm{CaTiO}_{3}$ has phonons in this frequency range, too. ${ }^{46}$ Therefore, we believe this line should be attributed to the phonons which are not localized within thin $\mathrm{SrTiO}_{3}, \mathrm{BaTiO}_{3}$ or $\mathrm{CaTiO}_{3}$ layers. These vibrations likely involve the atoms of the neighboring layers of another materials, so they belong to the whole superlattice. This is also the case for the $\mathrm{LO}_{3}$ mode, observed at about $485 \mathrm{~cm}^{-1}$, which has nearly the same frequency in these materials. The peak of $\mathrm{TO}_{4}$ phonon observed at $\sim 540-545 \mathrm{~cm}^{-1}$, likely has a contribution from all three layers, too. The frequencies of this phonon in bulk $\mathrm{BaTiO}_{3}$ $\mathrm{SrTiO}_{3}$ and $\mathrm{CaTiO}_{3}$ are also close $\left(522,545\right.$ and $547 \mathrm{~cm}^{-1}$, respectively), and can be even closer in our heterostructures due to strain.

The broad feature observed in the range $700-800 \mathrm{~cm}^{-1}$ is assigned to the $\mathrm{LO}_{4}$ phonons. In bulk $\mathrm{BaTiO}_{3}$ this feature is seen at about $720 \mathrm{~cm}^{-1}$, while in $\mathrm{SrTiO}_{3}{ }^{44}$ and $\mathrm{CaTiO}_{3}{ }^{46}$ the frequencies are significantly higher, about $795 \mathrm{~cm}^{-1}$. Therefore, this phonon can be considered as localized either in $\mathrm{BaTiO}_{3}$ or in $\mathrm{SrTiO}_{3}$ and $\mathrm{CaTiO}_{3}$ layers. The spectral line is broad, so the contributions of $\mathrm{BaTiO}_{3}$ and $\mathrm{SrTiO}_{3}+\mathrm{CaTiO}_{3}$ layers are hard to distinguish, but one can see that the peak shifts towards lower frequencies in SLs with larger $\mathrm{BaTiO}_{3}$ content.

As the thickness of $\mathrm{BaTiO}_{3}$ layers increases, they become partially relaxed, and the spectra closely resemble that of single $\mathrm{BaTiO}_{3}$ film (compare two bottom spectra in Fig.1). The spectra of the SLs studied contain no clear indication of the presence of the soft $E$ modes. (The low-frequency cut-off of the spectra was at $40 \mathrm{~cm}^{-1}$ We were unable to measure closer to the laser line because of high stray light background.)

Figure 2 shows the Raman spectra as a function of temperature for the C2B4S2 SL. Other superlattices exhibit similar temperature evolution of Raman spectra. From these spectra the $T_{c}$ can be determined. Bulk crystalline $\mathrm{SrTiO}_{3}$ and $\mathrm{CaTiO}_{3}$ are paraelectric at all temperatures. Bulk crystalline $\mathrm{BaTiO}_{3}$ is cubic and paraelectric above $T_{c}=403 \mathrm{~K}$, becomes tetragonal and ferroelectric below $T_{c}$, and goes through additional transitions to 
This is an author-produced, peer-reviewed version of this article. The final, definitive version of this document can be found online

at Journal of Applied Physics, published by American Institute of Physics. Copyright restrictions may apply. doi: 10.1063/1.3087611

orthorhombic at $278 \mathrm{~K}$ and rhombohedral at $183 \mathrm{~K} \cdot{ }^{47}$ Each of the three ferroelectric phases of bulk $\mathrm{BaTiO}_{3}$ can be identified by its Raman spectra. ${ }^{41}$. As can be seen in Fig. 2, the positions and lineshapes of phonons in SLs remain nearly unchanged as the temperature increases. Therefore, the SLs remain in the single ferroelectric phase and the low-temperature phase transitions characteristic for bulk $\mathrm{BaTiO}_{3}$ are suppressed in the SLs studied. Biaxial compressive strain in $\mathrm{BaTiO}_{3}$ layers, which stabilizes the tetragonal $c$ phase, is likely cause for such a behavior, which was also observed in binary $\mathrm{BaTiO}_{3} / \mathrm{SrTiO}_{3} \mathrm{SLs}^{35}$

The $T_{c}$ determination from Raman spectra is based on the fact that cubic centrosymmetric perovskite-type crystals have no first-order Raman active modes in paraelectric phase. Therefore, Raman intensity of the superlattice phonons decreases with increasing temperature and disappear upon transition into paraelectric phase. Above the $T_{c}$ the spectra contain only the broad second order features. By plotting the first-order Raman intensity as a function of temperature, one can determine $T_{c}$, as the temperature where the intensity becomes zero. ${ }^{35}$. This is illustrated in Figure 3 for three of the SLs studied: S1B1C1, S2B4C2, and S1B3C2. We used the intensities of the $\mathrm{TO}_{2}$ and $\mathrm{TO}_{4}$ phonon lines (marked by arrows in Fig.2), because they do not overlap with the second-order features. The intensities are normalized by the Bose factor $n+1=(1-\exp (-\hbar \omega / k T))^{-1}$ (where $\hbar$ is Planck's constant, $\omega$ is phonon frequency, $k$ is Boltzmanns constant, and $T$ is temperature), and divided by the intensity of the corresponding mode at low temperatures $(30 \mathrm{~K})$. Both $\mathrm{TO}_{2}$ and $\mathrm{TO}_{4}$ phonons show similar behaviors and the dashed-dotted lines are linear fits to the average of the two modes. The linear fit corresponds to a parabolic decrease of polarization with temperature as Raman intensity is proportional to the square of atomic displacement. The $T_{c}$ of the sample is determined as the intersection of a dash-dotted line with the horizontal axis.

In the SLs studied a structural asymmetry is introduced by the presence of the three different layers, $\mathrm{BaTiO}_{3}, \mathrm{SrTiO}_{3}$, and $\mathrm{CaTiO}_{3}$, in each period. Therefore, as temperature reaches $T_{c}$, the phonon peaks should not disappear from the spectra completely. Raman intensity should rather drop to some small but non-zero value. However, this inversion symmetry breakdown appears to have a small effect in terms of atomic displacement patterns associated with phonons, and this residual above- $T_{c}$ Raman intensity appears too small to be detected. Therefore, the observed temperature evolution of Raman intensities shows a behavior similar to that of symmetric two-component SLs. ${ }^{35}$ 
This is an author-produced, peer-reviewed version of this article. The final, definitive version of this document can be found online

at Journal of Applied Physics, published by American Institute of Physics. Copyright restrictions may apply. doi: 10.1063/1.3087611

Figure 4 summarizes the results of the $T_{c}$ determination for all the SLs measured as a function of the thickness ratio $R=\frac{d_{B T}}{\left(d_{S T}+d_{C T}\right)}$, where $d_{B T}, d_{S T}$, and $d_{C T}$ are the thicknesses of $\mathrm{BaTiO}_{3}, \mathrm{SrTiO}_{3}$, and $\mathrm{CaTiO}_{3}$ layers, respectively. Even the $\mathrm{S} 1 \mathrm{~B} 1 \mathrm{C} 1$ superlattice, containing only one monolayer of ferroelectric material, $\mathrm{BaTiO}_{3}$, per period, is ferroelectric with $T_{c}$ close to room temperature $\left(283 \mathrm{~K}\right.$, as determined from Raman data). As one can see, $T_{c}$ increases significantly with increasing fraction of $\mathrm{BaTiO}_{3}$, provided that it remains fully (or nearly fully) strained. Effect of strain relaxation is demonstrated by the rightmost point in the Fig.4. It corresponds to the sample with the largest $\mathrm{BaTiO}_{3}$ fraction $(\mathrm{S} 2 \mathrm{~B} 8 \mathrm{C} 2)$, but it is partially relaxed, therefore $T_{c}$ decreases significantly. These results correlate with the data on ferroelectric polarization in these SLs. As reported by Lee et al., ${ }^{2}$ the strongest polarization enhancement was also observed in fully strained SLs with the largest ratio $R$.

\section{SUMMARY}

Ultraviolet Raman spectroscopy was applied to studies of three-component $\mathrm{BaTiO}_{3} / \mathrm{SrTiO}_{3} / \mathrm{CaTiO}_{3}$ superlattices grown by atomic-scale-precision pulsed laser deposition. Raman data show that even the superlattices having the ferroelectric $\mathrm{BaTiO}_{3}$ layers as thin as one unit cell are ferroelectric with $T_{c}$ near room temperature. The temperature evolution of Raman spectra indicates that all superlattices remain in the tetragonal ferroelectric phase with out-of-plane polarization in the entire temperature range below $T_{c}$. The latter was determined from the temperature dependence of the intensity of the first-order phonon peaks in Raman spectra. Significant variation of $T_{c}$ was observed; the superlattices with the highest thickness of ferroelectric $\mathrm{BaTiO}_{3}$ layers respective to to non-ferroelectric $\mathrm{SrTiO}_{3}$ and $\mathrm{CaTiO}_{3}$ layers have the highest $T_{c}$, provided that $\mathrm{BaTiO}_{3}$ layers remain highly strained. Strain relaxation causes a drastic decrease in the ferroelectric phase transition temperature.

This work was partially supported by the National Science Foundation (Grant DMR0705127), the US Department of Energy (Grant DE-FG02-01ER45907), DOE EPSCoR (Grant DE-FG02-04ER46142), and by Research Corporation for Science Advancement (Grant No.7134). H. N. L. was sponsored by the Division of Materials Sciences and En- 
This is an author-produced, peer-reviewed version of this article. The final, definitive version of this document can be found online

gineering, U.S. Department of Energy.

* Electronic address: dmitritenne@boisestate.edu

1 D. G. Schlom, J. H. Haeni, J. Lettieri, C. D. Theis, W. Tian, J. C. Jiang, and X. Q. Pan, Mater. Sci. Eng. B 87, 282 (2001).

2 H. N. Lee, H. M. Christen, M. F. Chisholm, C. M. Rouleau, and D. H. Lowndes, Nature 433, $395(2005)$.

3 A.-B. Posadas, M. Lippmaa, F. J. Walker, M. Dawber, C. H. Ahn, and J.-M. Triscone, in Physics of Ferroelectrics: A Modern Perspective, edited by K. M. Rabe, C. H. Ahn, and J.-M. Triscone (Springer, Berlin, 2007), p. 219.

4 C. H. Ahn, K. M. Rabe, and J.-M. Triscone, Science 303, 488 (2004).

5 Nanoscale Phenomena in Ferroelectric Thin Films, edited by Seungbum Hong (Springer, Berlin, 2004)

6 M. Dawber, K. M. Rabe, and J. F. Scott, Rev. Mod. Phys. 77, 1083 (2005).

7 A. Rüdiger, T. Schneller, A. Roelofs, S. Tiedke, T. Schmitz, and R. Waser, Appl. Phys. A 80, $1247(2005)$

8 C. Lichtensteiger, M. Dawber, and J.-M. Triscone, in Physics of Ferroelectrics: A Modern Perspective, edited by K. M. Rabe, C. H. Ahn, and J.-M. Triscone (Springer, Berlin, 2007), p. 305.

9 M. Dawber, N. Stucki, C. Lichtensteiger, S. Gariglio, P. Ghosez, and J.-M. Triscone, Adv. Mater. 19, 4153 (2007)

10 H. Tabata, H. Tanaka, and T. Kawai, Appl. Phys. Lett. 65, 1970 (1994)

11 T. Shimuta, O. Nakagawara, T. Makino, S. Arai, H. Tabata, and T. Kawai, J. Appl. Phys. 91, $2290(2002)$.

12 F. Le Marrec, R. Farhi, M. El Marssi, J. L. Dellis, M. G. Karkut, and D. Ariosa, Phys. Rev. B 61, R6447 (2000).

13 J. C. Jiang, X. Q. Pan, W. Tian, C. D. Theis, and D. G. Schlom, Appl. Phys. Lett. 74, 2851 (1999).

14 M. Dawber, C. Lichtensteiger, M. Cantoni, M. Veithen, P. Ghosez, K. Johnston, K. M. Rabe, and J.-M. Triscone, Phys. Rev. Lett. 95, 177601 (2005). 
This is an author-produced, peer-reviewed version of this article. The final, definitive version of this document can be found online

15 E. Bousquet, M. Dawber, N. Stucki, C. Lichtensteiger, P. Hermet, S. Gariglio, J.-M. Triscone, and P. Ghosez, Nature 452, 732 (2008).

16 H. M. Christen, L. A. Boatner, J. D. Budai, M. F. Chisholm, L. A. Gea, P. J. Marrero, D. P. Norton, Appl. Phys. Lett. 68, 1488 (1996)

17 J. Sigman, D. P. Norton, H. M. Christen, P. H. Fleming, and L. A. Boatner, Phys. Rev. Lett., 88, 097601 (2002).

18 H. M. Christen, E. D. Specht, S. S. Silliman, and K. S. Harshavardhan, Phys. Rev. B 68, 020101(R) (2003).

19 M. P.Warusawithana, E. V. Colla, J. N. Eckstein, and M. B.Weissman, Phys. Rev. Lett. 90, $036802(2003)$.

20 J. B. Neaton and K. M. Rabe, Appl. Phys. Lett. 82, 1586 (2003)

21 S. M. Nakhmanson, K. M. Rabe, and D. Vanderbilt, Appl. Phys. Lett. 87, 102906 (2005).

22 S. M. Nakhmanson, K. M. Rabe, and D. Vanderbilt, Phys. Rev. B 73, 060101(R) (2006).

23 D. G. Schlom, L.-Q. Chen, C.-B. Eom, K. M. Rabe, S. K. Streiffer, and J.-M. Triscone, Annu. Rev. Mater. Res. 37, 589 (2007), and references therein.

24 K. J. Choi, M. Biegalski, Y. L. Li, A. Sharan, J. Schubert, R. Uecker, P. Reiche, Y. B. Chen, X. Q. Pan, V. Gopalan, L.-Q. Chen, D. G. Schlom, and C. B. Eom, Science 306, 1005 (2004).

25 J. H. Haeni, P. Irvin, W. Chang, R. Uecker, P. Reiche, Y. L. Li, S. Choudhury, W. Tian, M. E. Hawley, B. Craigo, A. K. Tagantsev, X. Q. Pan, S. K. Streiffer, L. Q. Chen, S. W. Kirchoefer, J. Levy, and D. G. Schlom, Nature 430, 758 (2004).

26 N. A. Spaldin, Science 304, 1606 (2004).

27 M. E. Lines and A. M. Glass, Principles and Applications of Ferroelectrics and Related Materials (Oxford University Press, New York 1977).

28 T. Tybell, C. H. Ahn, and J.-M. Triscone, Appl. Phys. Lett. 75, 856 (1999).

29 J. Junquera and P. Ghosez, Nature 422, 506 (2003).

30 N. Sai, A. M. Kolpak, and A. M. Rappe, Phys. Rev. B 72, 020101(R) (2005).

31 D. D. Fong, G. B. Stephenson, S. K. Streiffer, J. A. Eastman, O. Auciello, P. H. Fuoss, and C. Thompson, Science 304, 1650 (2004).

32 C. Lichtensteiger, J.-M. Triscone, J. Junquera, and P. Ghosez, Phys. Rev. Lett. 94, 047603 (2005).

33 L. Despont, C. Koitzsch, F. Clerc, M. G. Garnier, P. Aebi, C. Lichtensteiger, J.-M. Triscone, 
This is an author-produced, peer-reviewed version of this article. The final, definitive version of this document can be found online

F. J. Garcia de Abajo, E. Bousquet, and Ph. Ghosez, Phys. Rev. B 73, 094110 (2006).

34 D. D. Fong, A. M. Kolpak, J. A. Eastman, S. K. Streiffer, P. H. Fuoss, G. B. Stephenson, Carol Thompson, D. M. Kim, K. J. Choi, C. B. Eom, I. Grinberg, and A. M. Rappe Phys. Rev. Lett. 96, 127601 (2006).

D. A. Tenne, A. Bruchhausen, N. D. Lanzillotti-Kimura, A. Fainstein, R. S. Katiyar, A. Cantarero, A. Soukiassian, V. Vaithyanathan, J. H. Haeni, W. Tian, D. G. Schlom, K. J. Choi, D. M. Kim, C. B. Eom, H. P. Sun, X. Q. Pan, Y. L. Li, L. Q. Chen, Q. X. Jia, S. M. Nakhmanson, K. M. Rabe, and X. X. Xi, Science 313, 1614 (2006).

36 N. Sai, B. Meyer, and D. Vanderbilt, Phys. Rev. Lett. 84, 5636 (2000).

37 W. Cochran, Adv. Phys. 9, 387 (1960).

38 H. N. Lee, H. M. Christen, M. F. Chisholm, C. M. Rouleau, and D. H. Lowndes, Appl. Phys. Lett. 84, 4107 (2004).

39 A. Vailionis, W. Siemons, and G Koster, Appl. Phys. Lett. 93, 051909 (2008).

40 A. Scalabrin, A. S. Chaves, D. S. Shim, and S. P. S. Porto, Phys. Status Solidi B 79, 731 (1977).

41 D. A. Tenne, X. X. Xi, Y. L. Li, L. Q. Chen, A. Soukiassian, M. H. Zhu, A. R. James, J. Lettieri, D. G. Schlom, W. Tian, and X. Q. Pan, Phys. Rev. B 69, 174101 (2004).

42 W. G. Nilsen and J. G. Skinner, J. Chem. Phys. 48, 2240 (1968).

43 A. A. Sirenko, I. A. Akimov, J. R. Fox, A. M. Clark, Hong-Cheng Li, Weidong Si, and X. X. Xi, Phys. Rev. Lett. 82, 4500 (1999).

44 I. A. Akimov, A. A. Sirenko, A. M. Clark, J.-H. Hao, and X. X. Xi, Phys. Rev. Lett. 84, 4625 (2000).

45 P. McMillan and N. Ross, Phys. Chem. Miner. 16, 21 (1988).

46 V. Železný, E. Cockayne, J. Petzelt, M. F. Limonov, D. E. Usvyat, V. V. Lemanov, and A. A. Volkov, Phys. Rev. B 66, 224303 (2002).

47 Landolt-Börnstein: Numerical Data and Functional Relationships in Science and Technology, New Series. Group III, Volume 36, Subvolume A1, edited by Y. Shiozaki, E. Nakamura and T. Mitsui (Springer, Berlin, 2001), p. 67. 
This is an author-produced, peer-reviewed version of this article. The final, definitive version of this document can be found online at Journal of Applied Physics, published by American Institute of Physics. Copyright restrictions may apply. doi: 10.1063/1.3087611

TABLE I: Parameters of the superlattice samples studied

\begin{tabular}{lcccc}
\hline Sample & Structure & $\frac{d_{B T}}{d_{S T}+d_{C T}}$ & Strain & $T_{c}$ from \\
label & ratio & relaxation & Raman \\
& & & $(\%)$ & $(\mathrm{K})$ \\
\hline S1B1C1 & $\left(\mathrm{CaTiO}_{3}\right)_{1} /\left(\mathrm{BaTiO}_{3}\right)_{1} /\left(\mathrm{Sr} \mathrm{TiO}_{3}\right)_{1}$ & 0.5 & 0 & 283 \\
S4C2B4C2 & $\left(\mathrm{CaTiO}_{3}\right)_{2} /\left(\mathrm{BaTiO}_{3}\right)_{4} /\left(\mathrm{CaTiO}_{3}\right)_{2} /\left(\mathrm{SrTiO}_{3}\right)_{4}$ & 0.5 & 0 & 389 \\
C2B4S2 & $\left(\mathrm{SrTiO}_{3}\right)_{2} /\left(\mathrm{BaTiO}_{3}\right)_{4} /\left(\mathrm{CaTiO}_{3}\right)_{2}$ & 1.0 & 0 & 447 \\
S2B4C2 & $\left(\mathrm{CaTiO}_{3}\right)_{2} /\left(\mathrm{BaTiO}_{3}\right)_{4} /\left(\mathrm{SrTiO}_{3}\right)_{2}$ & 1.0 & 0 & 453 \\
S1B3C1 & $\left(\mathrm{CaTiO}_{3}\right)_{1} /\left(\mathrm{BaTiO}_{3}\right)_{3} /\left(\mathrm{SrTiO}_{3}\right)_{1}$ & 1.5 & 0.08 & 493 \\
S2B6C2 & $\left(\mathrm{CaTiO}_{3}\right)_{2} /\left(\mathrm{BaTiO}_{3}\right)_{6} /\left(\mathrm{SrTiO}_{3}\right)_{2}$ & 1.5 & 0.05 & 488 \\
S2B8C2 & $\left(\mathrm{CaTiO}_{3}\right)_{2} /\left(\mathrm{BaTiO}_{3}\right)_{8} /\left(\mathrm{SrTiO}_{3}\right)_{2}$ & 2.0 & 1.21 & 438 \\
\hline
\end{tabular}




\section{FIGURE CAPTIONS}

FIG. 1: (Color online) Low-temperature Raman spectra for $\left(\mathrm{CaTiO}_{3}\right) /\left(\mathrm{BaTiO}_{3}\right) /\left(\mathrm{SrTiO}_{3}\right)$ superlattices and a $200 \mathrm{~nm}$-thick $\mathrm{BaTiO}_{3}$ film at $30 \mathrm{~K}$.

FIG. 2: (Color online) Temperature evolution of Raman specrta for $\left(\mathrm{SrTiO}_{3}\right)_{2} /\left(\mathrm{BaTiO}_{3}\right)_{4} /\left(\mathrm{CaTiO}_{3}\right)_{2}$ superlattice. Arrows indicate Raman peaks used for $T_{c}$ determination.

FIG. 3: (Color online) Temperature dependencies of normalized Raman intensities of $\mathrm{TO}_{2}$ (solid symbols) and $\mathrm{TO}_{4}$ (open symbols) phonons for three $\left(\mathrm{CaTiO}_{3}\right)_{2} /\left(\mathrm{BaTiO}_{3}\right)_{4} /\left(\mathrm{SrTiO}_{3}\right)_{2}$ superlattices. The dash-dotted lines are fits to a linear temperature dependence.

FIG. 4: Results of $T_{c}$ determination from Raman data for all superlattices studied. $T_{c}$ is plotted as a function of the thickness ratio $\frac{d_{B T}}{\left(d_{S T}+d_{C T}\right)}$. Dashed line is guide to an eye. 


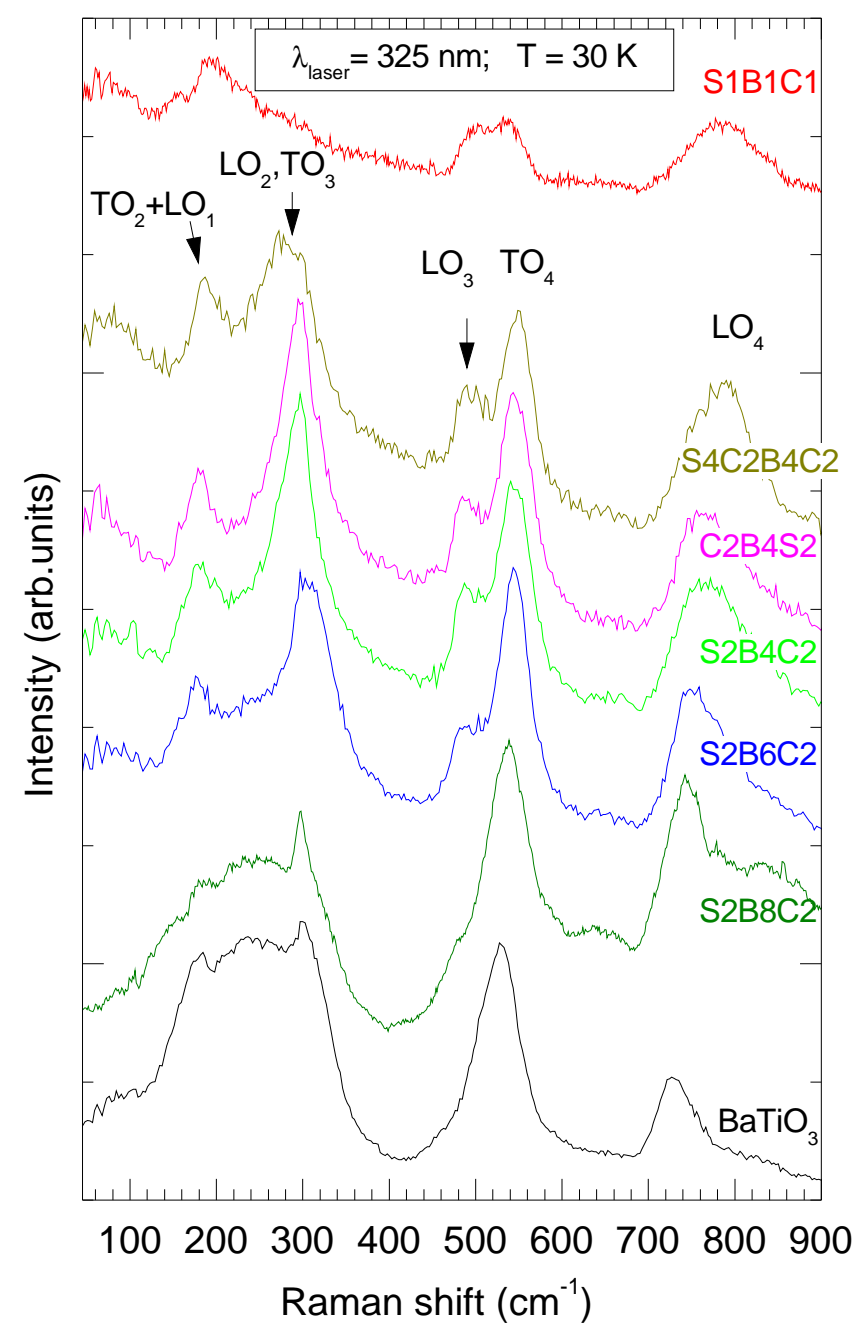

Figure 1 


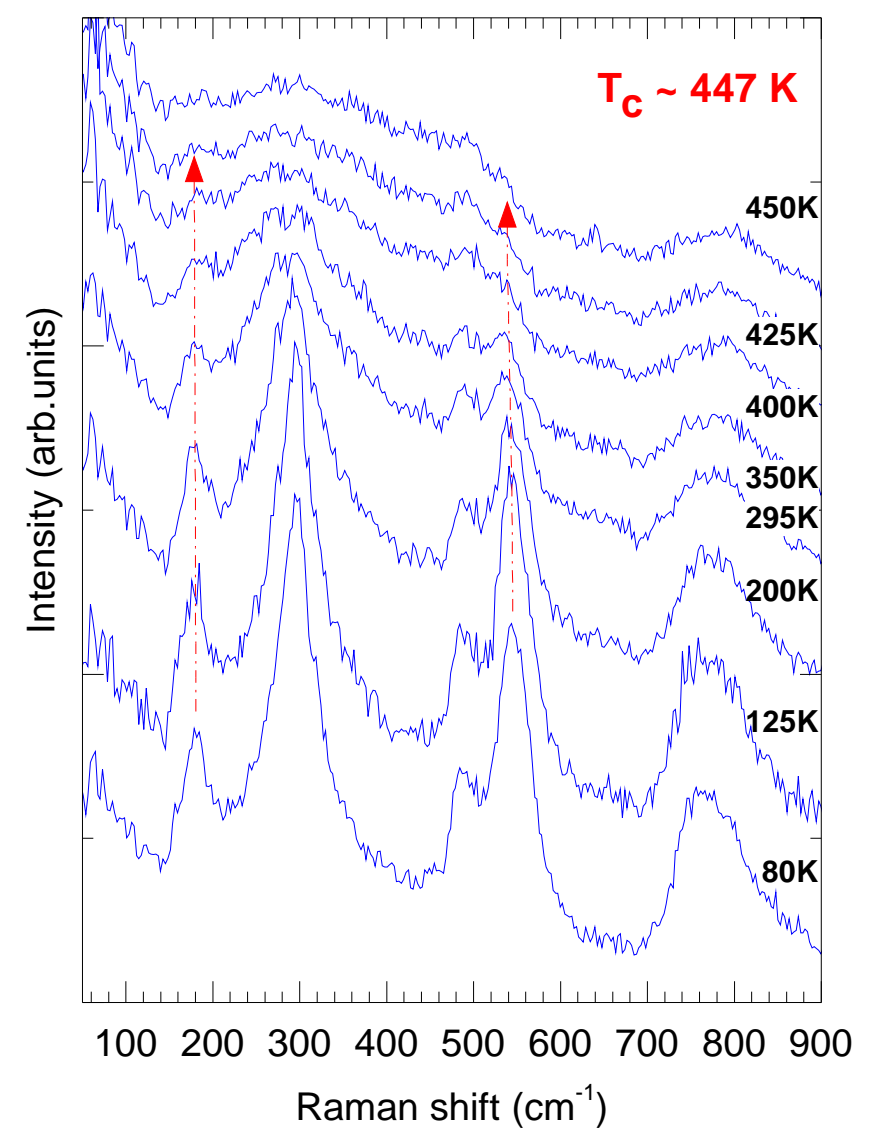

Figure 2 


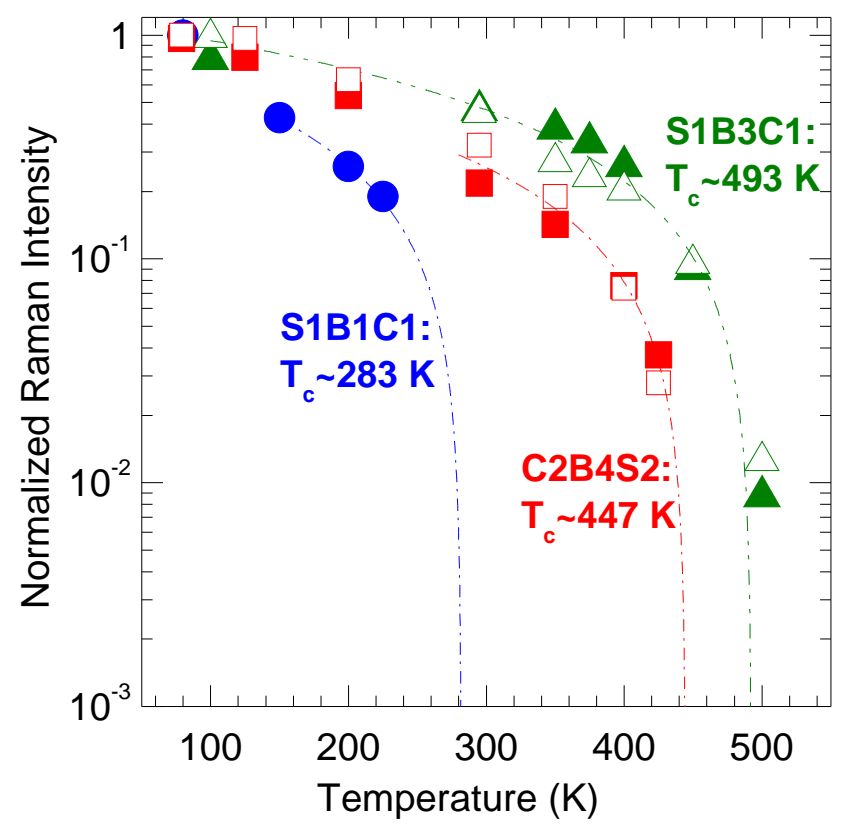

Figure 3 


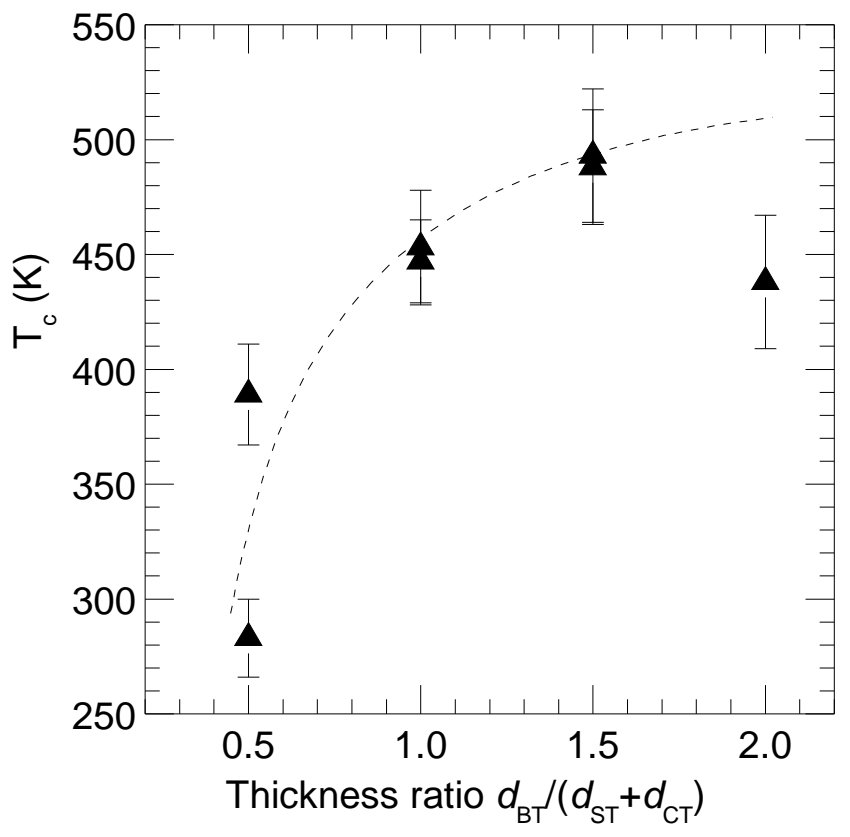

Figure 4 